methylphenidate in $63 \%$ of students, family practitioners in $17 \%$, psychiatrists, $11 \%$, and nurse practitioners in $3 \%$. When drugs other than methylphenidate were prescribed, the psychiatrist's role was increased to 29\%. (Safer DJ, Malever M. Stimulant treatment in Maryland Public Schools. Pediatrics September 2000;106:533-539). (Reprints: Daniel J Safer MD, 7702 Dunmanway, Baltimore, MD 21222).

COMMENT. In the authors' summary of their findings, the rate of medication, mainly methylphenidate, administered to students during school hours for ADHD in Maryland public schools was found to vary with several factors: 4-fold by gender (male:female ratio); 2-fold by ethnicity/race (white vs minority); 3-fold by school level (elementary vs high school); 6-fold by educational category (special vs regular); and 5 -fold by school district (highest vs lowest rate). Surveys of the prevalence of stimulant use in school age children must necessarily include all these variables. In addition, the specialty of the practitioner treating these patients must be considered, since the majority were supervised by pediatricians. The absence of the pediatric neurologist as a specialist for the treatment of ADHD in Maryland was disappointing.

\title{
ABNORMAL CEREBRAL BLOOD FLOW IN CHILDHOOD AUTISM
}

Brain-perfusion single photon emission computed tomography was used to determine regional cerebral blood flow (rCBF) in 23 children with infantile autism and 26 non-autistic controls, evaluated at the National Center of Neurology and Psychiatry, Kodaira City, Tokyo, Japan. Decreases in rCBF were identified in the bilateral insula, superior temporal gyri, and the left prefrontal cortices of autistic patients, compared to controls. Patients were divided into two clinical syndrome categories according to symptoms, and each was correlated with a specific pattern of perfusion in the limbic system and the medial prefrontal cortex. Category 1, with impairments in communication and social interaction, had altered perfusion in the medial prefrontal cortex and anterior cingulate gyrus, and category 2, with obsessive desire for sameness, had altered perfusion in the right medial temporal lobe. Perfusion abnormalities showed some relation to cognitive dysfunction, abnormal responses to sensory stimuli, and an obsessive desire for sameness. The patterns of perfusion point to possible locations of brain dysfunction that correlate with abnormal behavior in autistic individuals. (Ohnishi T, Matsuda H, Hashimoto $\mathrm{T}$ et al. Abnormal regional cerebral blood flow in childhood autism. Brain September 2000;123:1838-1844). (Respond: Dr Takashi Ohnishi, Department of Radiology, 4-1-1 Ogawa higashi, Kodaira City, Tokyo, Japan 187$0031)$.

COMMENT. Children with infantile autism have abnormalities of cerebral perfusion involving the insula, temporal cortex, medial prefrontal cortex, anterior cingulate gyrus and hippocampus. These perfusion abnormalities are related to the cognitive and behavioral abnormalities seen in autism, and correlate with specific locations of brain dysfunction.

The authors refer to a deficit in the theory of mind tasks (ToM), a widely accepted explanation for the cognitive disorders of communication, socialization, and imagination in autistic children. ToM is the ability to explain and predict the behavior of others in terms of their mental states, such as beliefs and desires. Studies show that most autistic subjects fail the so-called false-belief task, an indication of a central cognitive deficit. The ability to attribute mental states has been located in the medial prefrontal cortex, an area with altered cerebral blood flow in autism. The orbitofrontal cortex and amygdala have also been associated with the ToM and ability to attribute mental states (Baron-Cohen et al. 1999). 